\title{
Estimating the quality-of-life-adjusted gap time distribution of successive events subject to censoring
}

\author{
BY ADIN-CRISTIAN ANDREI AND SUSAN MURRAY \\ Department of Biostatistics, University of Michigan, 1420 Washington Heights, Ann Arbor, \\ Michigan 48109, U.S.A. \\ andreia@umich.edu skmurray@umich.edu
}

\begin{abstract}
SUMMARY
When treatment effects are studied in the context of successive or recurrent life events, separate analyses of the quality-of-life scores and of the inter-event, gap, times might lead to possibly contradictory conclusions. In an attempt to reconcile this, we propose a unitary and more comprehensive nonparametric analysis that combines the two separate analyses by introducing the quality-of-life-adjusted gap time concept. Inverse probability of censoring estimators of the quality-of-life-adjusted gap time joint and conditional distributions are proposed and are shown to be consistent and asymptotically normal. Simulations performed in a variety of scenarios indicate that the joint and conditional quality-of-life-adjusted gap time distribution estimators are virtually unbiased, with properly estimated standard errors and asymptotic normality features. An example from the International Breast Cancer Study Group Trial V illustrates the use of the proposed estimators.
\end{abstract}

Some key words: Gap time; Inverse weighting; Nonparametric; Quality-of-life; Recurrent; Survival.

\section{INTRODUCTION}

During a clinical trial one may experience improvement or deterioration in quality-oflife. It is often of interest to describe both the magnitude of the overall quality-of-life changes and the way quality-of-life evolves during a series of events of the same type, called recurrent, or of different types, called successive. Certainly, the timing of adverse repetitive events also influences the quality-of-life at a basic level.

Repeated hospitalisations or pulmonary exacerbations are examples of recurrent event occurrences in chronic diseases, whereas a series of different-type events such as therapy initiation time, end of the toxicity period, end of the disease-free period and death illustrate types of successive event. Regardless of the event nature, the inter-event times are usually referred to as gap times. Although quality-of-life scores and gap times are usually analysed separately, each analysis fails to take into account all the aspects influencing quality-oflife. A comprehensive summary of both sources of information would be particularly useful in studying chronic or other diseases subject to depreciating quality-of-life scores but extended lifetimes.

As an attempt to reconcile quality- and quantity-of-life through a series of life events, we propose the quality-adjusted gap time concept, having in mind a scenario in which a 
deteriorating trend in quality-of-life is observed, leading to a sequence of recurrent or successive adverse events. Analyses pertaining to 'average' gap times in this context might convey a highly incomplete image of one individual's health state, since quality-of-life adjustments are likely to vary as the disease progresses. Therefore, quality-of-life gapadjustment emerges as a natural undertaking in the context of nonparametric statistical inference. Although in the recurrent events literature it is common to assume that the gap times are conditionally independent and identically distributed (Wang et al., 2001), our approach does not require such assumptions.

The quality-of-life-adjusted gap time concept is particularly appealing in a cancer clinical trial like the International Breast Cancer Study Group Trial V, where increased amounts of chemotherapy may lead to lower quality-of-life during the toxicity period, but may have a positive impact on the quality-of-life during the cancer-relapse period. Understanding the joint distribution of the quality-adjusted gap times for landmark events during the trial would be useful. It would enable the investigator to provide the patient with a wide spectrum of 'chemotherapy dose during the toxicity period versus life prolongation at superior quality-of-life levels during disease relapse' options, in terms of both joint and conditional probability statements.

Zhao \& Tsiatis $(1997,1999)$ propose consistent estimators for the quality-adjusted lifetime, while 'recognising that there is still debate on the use of such a simple measure'. However, the quality-adjusted lifetime concept is favourably regarded and its use is advocated in clinical practice, especially through derivatives such as 'time without symptoms and toxicity' or 'quality-adjusted time without symptoms and toxicity'; see for example Gelber et al. (1989). Huang \& Louis (1999) study mean quality-adjusted lifetime estimation, while van der Laan \& Hubbard (1999) propose doubly-robust quality-adjusted lifetime estimators under dependent censoring.

Even under independent or random censoring, all but the first gap time are subject to induced dependent censoring, because of dependence among the recurrent or successive events, as pointed out for example by Lin et al. (1999). Various nonparametric estimators of the (joint) gap time(s) distribution have been proposed by, among others, Huang \& Louis (1998), Wang \& Wells (1998), Lin et al. (1999), Wang et al. (2001), Wang \& Chang (1999), Huang (2000), Peña et al. (2001) and van der Laan et al. (2002). Some, but not all, of these methods involve inverse probability of censoring techniques as in Robins \& Rotnitzky (1992).

\section{Estimation OF THE JOINT AND CONDITIONAL DisTRIBUTIONS OF THE QUALITY-OF-LIFE-ADJUSTED GAP TIMES}

Since study entry, an individual may experience a number of consecutive events at continuously distributed times $Y_{1}<Y_{2}<Y_{3}<\ldots$. Suppose that the interest is centred upon the first $K$ such events, and from now on consider $K$ to be fixed. Define the inter-event or gap times as $T_{j}=Y_{j}-Y_{j-1}$, with $j=1, \ldots, K$ and $Y_{0}=0$. The total individual follow-up time may not be completely observed because of a censoring event $C$, which is assumed to be continuous and independent of the vector $\left(Y_{1}, Y_{2}, \ldots, Y_{K}\right)$. However, unless $Y_{j-1}$ and $T_{j}$ are independent, $T_{j}$ is dependently censored by $C-Y_{j-1}$, thereby creating the induced dependent censoring problem. Note that no restriction is imposed on the dependence structure of the inter-event times.

For an individual, let the health history be quantified by a continuous time stochastic process $V($.$) , whose states are \{0,1, \ldots, S\}$. Ordering with respect to disease severity is 
assumed among the health states, state ' $O$ ' representing death and state ' $S$ ' being perfect health. State ' 0 ' is assumed to be absorbing, while all others are transient. Let $Q($.) be a deterministic, nondecreasing and known utility function assigning to each health state a utility value between 0 , corresponding to state ' 0 ' and 1 , to state ' $S$ '. Although dependence is allowed and likely between $V($.$) and \left(Y_{1}, Y_{2}, \ldots, Y_{K}\right)$, it is assumed that $V($.$) and C$ are independent.

For reasons that will become clear later on, we assume the existence of two strictly positive constants $L$ and $\delta$ such that $Y_{1}<\ldots<Y_{K}<L$ and $\operatorname{pr}(C>L)>\delta>0$. In brief, we require that the probability of observing complete, uncensored data is strictly bounded away from zero. As a result of censoring, one observes instead

$$
\left\{\left(\tilde{Y}_{1}, \Delta_{1}\right),\left(\tilde{Y}_{2}, \Delta_{2}\right), \ldots,\left(\tilde{Y}_{K}, \Delta_{K}\right), V(t), t \leqslant \tilde{Y}_{K}\right\},
$$

where $\tilde{Y}_{j}=\min \left(Y_{j}, C\right)$ and $\Delta_{j}=I\left(Y_{j} \leqslant C\right)$, for $j=1, \ldots, K$.

Define the quality-adjusted event times to be

$$
Q Y_{j}=\int_{0}^{Y_{j}} Q\{V(t)\} d t
$$

and the quality-adjusted gap times to be

$$
Q T_{j}=\int_{Y_{j-1}}^{Y_{j}} Q\{V(t)\} d t .
$$

In the presence of censoring, their observed versions are, respectively,

$$
\tilde{Q} Y_{j}=\int_{0}^{\tilde{Y}_{j}} Q\{V(t)\} d t, \quad \widetilde{Q} T_{j}=\int_{\tilde{Y}_{j-1}}^{\tilde{Y}_{j}} Q\{V(t)\} d t \quad(j=1, \ldots, K) .
$$

Let $q^{(K-1)}, q^{(K)}, q^{\prime(K)}$ and $q_{0}^{(K)}$ be the vectors of nonnegative numbers $\left(q_{1}, q_{2}, \ldots, q_{K-1}\right)$, $\left(q^{(K-1)}, q_{K}\right),\left(q^{(K-1)}, q_{K}^{\prime}\right)$ and $\left(q^{(K-1)}, 0\right)$, respectively. Similarly, define $r^{(K-1)}, r^{(K)}, r^{\prime(K)}$ and $r_{0}^{(K)}$ in the obvious way, where $r_{j}$ replaces $q_{j}$, for $j=1, \ldots, K$. Let $T^{(K-1)}, T^{(K)}, Q T^{(K-1)}$ and $Q T^{(K)}$ denote the vectors $\left(T_{1}, T_{2}, \ldots, T_{K-1}\right),\left(T^{(K-1)}, T_{K}\right),\left(Q T_{1}, Q T_{2}, \ldots, Q T_{K-1}\right)$ and $\left(Q T^{(K-1)}, Q T_{K}\right)$, respectively.

Based on the observed data, the goal is to estimate the joint distribution

$$
F_{Q}\left(q^{(K)}\right)=\operatorname{pr}\left(Q T^{(K)} \leqslant q^{(K)}\right)
$$

and the conditional distribution $F_{Q}^{K \mid K-1}\left(q_{K} \mid q^{(K-1)}\right)=\operatorname{pr}\left(Q T_{K} \leqslant q_{K} \mid Q T^{(K-1)} \leqslant q^{(K-1)}\right)$. If we let

$$
H_{Q}\left(q^{(K)}\right)=\operatorname{pr}\left(Q T^{(K-1)} \leqslant q^{(K-1)}, Q T_{K}>q_{K}\right),
$$

then $F_{Q}\left(q^{(K)}\right)=H_{Q}\left(q_{0}^{(K)}\right)-H_{Q}\left(q^{(K)}\right)$ and $F_{Q}^{K \mid K-1}\left(q_{K} \mid q^{(K-1)}\right)=1-H_{Q}\left(q^{(K)}\right) / H_{Q}\left(q_{0}^{(K)}\right)$. Thus, to estimate both $F_{Q}($.$) and F_{Q}^{K \mid K-1}\left(. \mid\right.$.) it suffices to estimate $H_{Q}($.$) .$

We define $m\left(q_{K}\right)=\inf \left\{s \geqslant Y_{K-1} ; \int_{Y_{K-1}}^{s} Q\{V(t)\} d t \geqslant q_{K}\right\}$ and $D\left(q_{K}\right)=\min \left\{m\left(q_{K}\right), Y_{K}\right\}$. This expression states that $D\left(q_{K}\right)$ marks the first time during the unfolding of the $K$ th gap time that the individual has accumulated at least $q_{K}$ quality-adjusted time since the last event. Should this event not happen until time $Y_{K}, D\left(q_{K}\right)$ is assigned the value $Y_{K}$.

Throughout this presentation, attaching the index $i=1, \ldots, n$ indicates that the quantity in question is computed for the $i$ th individual. Without censoring, $H_{Q}\left(q^{K)}\right)$ could 
be consistently estimated by

$$
n^{-1} \sum_{i=1}^{n} I\left\{Q T_{i}^{(K-1)} \leqslant q^{(K-1)}, Q T_{K i}>q_{K}\right\}
$$

In the presence of censoring, an inverse probability-of-censoring weighted estimator can be constructed by carefully noting when the indicator function of interest is observed. First, if $\widetilde{Q} T_{K}>q_{K}>0$, then $\widetilde{Q} T_{1}, \widetilde{Q} T_{2}, \ldots, \tilde{Q} T_{K-1}$ are uncensored and $Y_{K-1}<D\left(q_{K}\right)<Y_{K}$. Consequently, if

$$
\begin{aligned}
& A\left(q^{(K)}\right)=I\left\{Q T^{(K-1)} \leqslant q^{(K-1)}, Q T_{K}>q_{K}, C>D\left(q_{K}\right)\right\}, \\
& B\left(q^{(K)}\right)=I\left\{\widetilde{Q} Y_{1} \leqslant q_{1}, \tilde{Q} Y_{2}-\tilde{Q} Y_{1} \leqslant q_{2}, \ldots, \tilde{Q} Y_{K-1}-\tilde{Q} Y_{K-2} \leqslant q_{K-1}, \tilde{Q} Y_{K}-\tilde{Q} Y_{K-1}>q_{K}\right\},
\end{aligned}
$$

then $A\left(q^{(K)}\right)=B\left(q^{(K)}\right)$. Then, if $G(u)=\operatorname{pr}(C>u)$ were known,

$$
\tilde{H}_{Q}\left(q^{(K)}\right)=n^{-1} \sum_{i=1}^{n} \frac{A_{i}\left(q^{(K)}\right)}{G\left\{D_{i}\left(q_{K}\right)\right\}}
$$

would be an unbiased estimator for $H_{Q}\left(q^{(K)}\right)$. To see why, note that

$$
\begin{aligned}
E\left[\frac{A\left(q^{(K)}\right)}{G\left\{D\left(q_{K}\right)\right\}}\right] & =E\left(E\left[\frac{A\left(q^{(K)}\right)}{G\left\{D\left(q_{k}\right)\right\}} \mid T^{(K)}, V(.)\right]\right) \\
& =E\left(I\left(Q T^{(K-1)} \leqslant q^{(K-1)}, Q T_{K}>q_{K}\right) E\left[\frac{I\left\{C \geqslant D\left(q_{K}\right)\right\}}{G\left\{D\left(q_{K}\right)\right\}} \mid T^{(K)}, V(.)\right]\right) \\
& =E\left\{I\left(Q T^{(K-1)} \leqslant q^{(K-1)}, Q T_{K}>q_{K}\right)\right\}=H_{Q}\left(q^{(K)}\right) .
\end{aligned}
$$

Since $G($.$) is unknown, it is estimated by the Kaplan-Meier estimator \hat{G}($.$) of the censoring$ time survival function, using $\left\{\left(\widetilde{Y}_{K i}, 1-\Delta_{K i}\right) ; i=1, \ldots, n\right\}$. Then a consistent estimator for $H_{Q}\left(q^{(K)}\right)$ is

$$
\hat{H}_{Q}\left(q^{(K)}\right)=n^{-1} \sum_{i=1}^{n} \frac{B_{i}\left(q^{(K)}\right)}{\widehat{G}_{\{}\left(D_{i}\left(q_{K}\right)\right\}},
$$

from which consistent estimators $\hat{F}_{Q}\left(q^{(K)}\right)$ and $\hat{F}_{Q}^{K \mid K-1}\left(q_{K} \mid q^{(K-1)}\right)$ of $F_{Q}\left(q^{(K)}\right)$ and $F_{Q}^{K \mid K-1}\left(q_{K} \mid q^{(K-1)}\right)$, respectively, can be constructed. The relevant asymptotic theory is developed in the Appendix.

\section{Simulation study}

Simulations have been conducted under two scenarios to assess the moderate sample size properties of $\hat{F}_{Q}\left(q_{1}, q_{2}\right)$ and $\hat{F}_{Q}^{2 \mid 1}\left(q_{2} \mid q_{1}\right)$, where $q_{1} \in\left\{\alpha_{1 ; 0 \cdot 25}, \alpha_{1 ; 0 \cdot 50}, \alpha_{1 ; 0 \cdot 75}\right\}$, $q_{2} \in\left\{\alpha_{2 ; 0 \cdot 25}, \alpha_{2 ; 0 \cdot 5}\right\}$ and $\alpha_{i ; r}$ is the $r$ th upper quantile of $Q T_{i}$, for $i=1,2$.

Correlated gap times $\left(T_{1}, T_{2}\right)$ have been generated from the $\operatorname{Ex}\left(\frac{1}{10}\right)$ and $\operatorname{Ex}\left(\frac{1}{6}\right)$ distributions, respectively, with between-gaps correlation levels $\rho$ successively being approximately 0 and $0 \cdot 3$. Throughout, we have employed the utility function $Q(s)=s / 100$, where $s$ belongs to the health states space $S=\{0,1, \ldots, 100\}$. Under the first simulation scenario, the health process was $V(s)=100$, for all $s \in S$, so that the quality-adjusted gap times $\left(Q T_{1}, Q T_{2}\right)$ were equal to their unadjusted counterparts $\left(T_{1}, T_{2}\right)$. For the second set of simulations, the value of the health history process $V($.$) at any time point between 0$ 
Table 1. Simulation results for $\hat{F}_{Q}\left(q_{1}, q_{2}\right)$ based on approximately $\rho$-correlated exponential successive gap times

\begin{tabular}{|c|c|c|c|c|c|c|c|c|c|c|}
\hline \multirow[b]{2}{*}{$\left(q_{1}, q_{2}\right)$} & \multicolumn{5}{|c|}{$\rho=0$} & \multicolumn{5}{|c|}{$\rho=0.3$} \\
\hline & $\mathrm{TP}$ & EP & SEP & MSE & $\mathrm{CP}$ & $\mathrm{TP}$ & EP & SEP & MSE & $\mathrm{CP}$ \\
\hline & \multicolumn{10}{|c|}{ Scenario 1} \\
\hline$(2 \cdot 877,1 \cdot 726)$ & $0 \cdot 062$ & 0.063 & $0 \cdot 018$ & $0 \cdot 018$ & 0.942 & 0.096 & 0.095 & $0 \cdot 021$ & $0 \cdot 021$ & 0.941 \\
\hline$(2 \cdot 877,4$ & $0 \cdot 125$ & $0 \cdot 125$ & 0.024 & $0 \cdot 024$ & 0.947 & $0 \cdot 164$ & $0 \cdot 164$ & 0.027 & 0.027 & $0 \cdot 945$ \\
\hline$(6.931,1$. & $\cdot 125$ & $0 \cdot 125$ & $0 \cdot 024$ & 0.025 & 0.949 & $0 \cdot 164$ & $0 \cdot 163$ & $0 \cdot 028$ & $0 \cdot 028$ & $0 \cdot 942$ \\
\hline 31,4 & $\cdot 250$ & $0 \cdot 250$ & 0.032 & 0.032 & 0.949 & $0 \cdot 298$ & $0 \cdot 298$ & 0.034 & $0 \cdot 034$ & 0.948 \\
\hline$(13 \cdot 863,1 \cdot 726)$ & $\cdot 187$ & $0 \cdot 188$ & 0.030 & $0 \cdot 030$ & $0 \cdot 949$ & $0 \cdot 217$ & $0 \cdot 216$ & $0 \cdot 031$ & $0 \cdot 031$ & 0.947 \\
\hline \multirow[t]{2}{*}{$(13 \cdot 863,4 \cdot 159)$} & 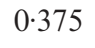 & $0 \cdot 376$ & $0 \cdot 038$ & 0.037 & $0 \cdot 946$ & $0 \cdot$ & $0 \cdot 412$ & 0.038 & $0 \cdot 038$ & $0 \cdot 945$ \\
\hline & \multicolumn{10}{|c|}{ rio 2} \\
\hline$(2 \cdot 3$ & .09 & 0.091 & $0 \cdot 021$ & $0 \cdot 02$ & 0.938 & $0 \cdot 122$ & $0 \cdot 122$ & $0 \cdot 024$ & $0 \cdot 024$ & 0.938 \\
\hline$(2 \cdot 308,2 \cdot 578)$ & $0 \cdot 168$ & $0 \cdot 168$ & $0 \cdot 028$ & 0.027 & $0 \cdot 942$ & $0 \cdot 201$ & $0 \cdot 201$ & 0.029 & 0.029 & 0.944 \\
\hline$(6 \cdot 231,1.009)$ & $0 \cdot 156$ & $0 \cdot 157$ & 0.027 & 0.027 & $0 \cdot 947$ & $0 \cdot 191$ & $0 \cdot 190$ & $0 \cdot 029$ & $0 \cdot 029$ & 0.947 \\
\hline$(6 \cdot 231,2 \cdot 578)$ & 0.299 & $0 \cdot 299$ & $0 \cdot 048$ & $0 \cdot 049$ & 0.948 & $0 \cdot 343$ & $0 \cdot 343$ & $0 \cdot 035$ & $0 \cdot 035$ & 0.948 \\
\hline$(13 \cdot 752,1.009)$ & $0 \cdot 208$ & $0 \cdot 210$ & $0 \cdot 031$ & $0 \cdot 031$ & $0 \cdot 945$ & $0 \cdot 231$ & $0 \cdot 230$ & $0 \cdot 031$ & $0 \cdot 031$ & 0.948 \\
\hline$(13 \cdot 752,2 \cdot 578)$ & $0 \cdot 408$ & 0.409 & 0.038 & 0.038 & 0.949 & 0.442 & $0 \cdot 441$ & 0.038 & 0.038 & 0.950 \\
\hline
\end{tabular}

TP, true probability $F_{Q}\left(q_{1}, q_{2}\right)$; EP, empirical mean of the $\hat{F}_{Q}\left(q_{1}, q_{2}\right)$ values; SEP, empirical standard error of the $\hat{F}_{Q}\left(q_{1}, q_{2}\right)$ values; MSE, empirical mean of estimated standard errors of $\hat{F}_{Q}\left(q_{1}, q_{2}\right)$ probabilities; CP, coverage probability of true $F_{Q}\left(q_{1}, q_{2}\right)$ by the $95 \%$ confidence intervals

and $T_{1}+T_{2}$ was found by linear interpolation, given that $V(0)=100$ :

$$
\begin{gathered}
V\left(T_{1}\right)= \begin{cases}100, & \text { if } T_{1} \geqslant t_{1 ; 0 \cdot 75}, \\
80, & \text { if } t_{1 ; 0 \cdot 5} \leqslant T_{1}<t_{1 ; 0 \cdot 75}, \\
60, & \text { if } t_{1 ; 0 \cdot 25} \leqslant T_{1}<t_{1 ; 0 \cdot 5}, \\
40, & \text { if } T_{1}<t_{1 ; 0 \cdot 25} ;\end{cases} \\
V\left(T_{1}+T_{2}\right)=V\left(T_{1}\right) \times \begin{cases}1 \cdot 0, & \text { if } T_{2} \geqslant t_{2 ; 0 \cdot 75}, \\
0 \cdot 9, & \text { if } t_{2 ; 0 \cdot 5} \leqslant T_{2}<t_{2 ; 0 \cdot 75}, \\
0 \cdot 8, & \text { if } t_{2 ; 0 \cdot 25} \leqslant T_{2}<t_{2 ; 0 \cdot 5}, \\
0 \cdot 7, & \text { if } T_{2}<t_{2 ; 0 \cdot 25} .\end{cases}
\end{gathered}
$$

In the definition of the health history process $V($.$) above, t_{i, r}$ was the $r$ th quantile of $T_{i}$, where $r \in\{0 \cdot 25,0 \cdot 50,0 \cdot 75\}$ and $i \in\{1,2\}$. Under this latter simulation scenario, with dependent $\left(Q T_{1}, Q T_{2}\right)$ quality-adjusted gaps being observed, we have captured a deteriorating trend in quality-of-life accompanied by shorter second gap times. Under each scenario, random samples of size $n=200$ were replicated 5000 times, with censoring, corresponding to end of follow-up, generated independently from $\operatorname{Un}(0,84)$. The results presented in Tables 1 and 2 confirm that the estimators $\widehat{F}_{Q}\left(q_{1}, q_{2}\right)$ and $\hat{F}_{Q}^{2 \mid 1}\left(q_{2} \mid q_{1}\right)$ are virtually unbiased in the case of finite sample sizes and the empirical standard errors of the estimated probabilities agree with the empirical means of the estimated standard errors. Also, the sample average coverage probabilities of the $95 \%$-confidence intervals for $F_{Q}\left(q_{1}, q_{2}\right)$ and $F_{Q}^{2 \mid 1}\left(q_{2} \mid q_{1}\right)$ are close to the nominal levels in all cases. 
Table 2. Simulation results for $\hat{F}_{Q}^{2 \mid 1}\left(q_{2} \mid q_{1}\right)$ based on approximately $\rho$-correlated exponential successive gap times

\begin{tabular}{|c|c|c|c|c|c|c|c|c|c|c|}
\hline \multirow[b]{2}{*}{$\left(q_{1}, q_{2}\right)$} & \multicolumn{5}{|c|}{$\rho=0$} & \multicolumn{5}{|c|}{$\rho=0 \cdot 3$} \\
\hline & ТР & EP & SEP & MSE & $\mathrm{CP}$ & $\mathrm{TP}$ & EP & SEP & MSE & $\mathrm{CP}$ \\
\hline & \multicolumn{10}{|c|}{ Scenario 1} \\
\hline$(2 \cdot 877,1 \cdot 726)$ & $0 \cdot 250$ & $0 \cdot 250$ & $0 \cdot 065$ & 0.064 & 0.940 & 0.384 & $0 \cdot 380$ & $0 \cdot 071$ & 0.071 & $0 \cdot 942$ \\
\hline$(2 \cdot 877,4 \cdot 159)$ & $0 \cdot 500$ & $0 \cdot 500$ & $0 \cdot 074$ & 0.074 & 0.939 & 0.654 & 0.655 & $0 \cdot 070$ & 0.070 & 0.942 \\
\hline$(6 \cdot 931,1 \cdot 726)$ & $0 \cdot 250$ & $0 \cdot 250$ & 0.032 & 0.032 & 0.944 & 0.327 & $0 \cdot 327$ & $0 \cdot 050$ & 0.049 & 0.943 \\
\hline$(6 \cdot 931,4 \cdot 159)$ & $0 \cdot 500$ & $0 \cdot 500$ & $0 \cdot 054$ & 0.053 & 0.944 & 0.596 & 0.597 & 0.052 & 0.052 & 0.945 \\
\hline$(13 \cdot 863,1 \cdot 726)$ & $0 \cdot 250$ & $0 \cdot 250$ & 0.038 & 0.038 & 0.948 & 0.289 & $0 \cdot 287$ & 0.039 & 0.039 & 0.947 \\
\hline \multirow[t]{2}{*}{$(13 \cdot 863,4 \cdot 159)$} & $0 \cdot 500$ & $0 \cdot 501$ & 0.044 & 0.044 & $0 \cdot 946$ & 0.552 & 0.550 & $0 \cdot 044$ & 0.044 & 0.947 \\
\hline & \multicolumn{10}{|c|}{ Scenario 2} \\
\hline$(2 \cdot 308,1 \cdot 009)$ & $0 \cdot 364$ & $0 \cdot 366$ & 0.072 & 0.071 & 0.942 & 0.489 & $0 \cdot 491$ & 0.073 & 0.073 & 0.947 \\
\hline$(2 \cdot 308,2 \cdot 578)$ & $0 \cdot 671$ & $0 \cdot 673$ & $0 \cdot 071$ & 0.071 & 0.936 & $0 \cdot 201$ & $0 \cdot 201$ & 0.030 & 0.029 & 0.944 \\
\hline$(6 \cdot 231,1 \cdot 009)$ & $0 \cdot 312$ & $0 \cdot 314$ & 0.048 & 0.049 & 0.948 & 0.381 & $0 \cdot 380$ & 0.051 & 0.051 & 0.947 \\
\hline$(6.231,2.578)$ & 0.598 & 0.598 & $0 \cdot 052$ & 0.052 & 0.949 & 0.686 & $0 \cdot 686$ & 0.049 & 0.049 & 0.947 \\
\hline$(13.752,1.009)$ & $0 \cdot 277$ & $0 \cdot 279$ & 0.039 & 0.039 & 0.952 & 0.308 & $0 \cdot 307$ & $0 \cdot 040$ & 0.040 & $0 \cdot 951$ \\
\hline$(13 \cdot 752,2 \cdot 578)$ & $0 \cdot 408$ & $0 \cdot 409$ & 0.038 & 0.038 & 0.949 & 0.589 & 0.589 & $0 \cdot 044$ & 0.043 & $0 \cdot 951$ \\
\hline
\end{tabular}

\section{International Breast Cancer Study Group Trial V example}

During the randomised adjuvant chemotherapy Trial $\mathrm{V}$ conducted by the International Breast Cancer Study Group (Gelber et al., 1992), 411 node-positive breast cancer patients received short-duration, 1-3 months, chemotherapy and 804 node-positive patients received long-duration, 6-7 months, chemotherapy. After a fully observed treatment toxicity period, patients experience a time without symptoms or toxicity, denoted by $T_{1}$, followed by a cancer relapse period, denoted by $T_{2}$, subject to censoring. Both $T_{1}$ and $T_{2}$ are measured in months. We base the current analyses on the first 108 months of follow-up.

It is of interest to know whether or not a longer quality-of-life-adjusted $T_{1}$ period, $Q T_{1}$, generally leads to a longer quality-of-life-adjusted $T_{2}$ period, $Q T_{2}$, in either of the two treatment arms. In order to understand better the implications stemming from the choice of the health process $V\left(\right.$.), we have envisaged two scenarios, $V_{1}($.$) and V_{2}($.$) . In either of$ them, $V($.$) assumes values in the health space S=\{0,1, \ldots, 100\}$ and the utility function is $Q(s)=s / 100$, for $s \in S$.

Under the first scenario, $V_{1}(t)=100$ on $\left[0, T_{1}\right)$ and $V_{1}(t)=50$ on $\left[T_{1}, T_{1}+T_{2}\right]$. Under the second scenario, $V_{2}(t)=100$ on $\left[0, T_{1}\right)$. If $T_{2}>84$ months, then $V_{2}(t)=100$ on $\left[T_{1}, T_{1}+T_{2}\right]$. Otherwise, if

$$
12 \times(6-i)<T_{2} \leqslant 12 \times(7-i) \quad(i=0,1, \ldots, 6),
$$

then $V_{2}\left(T_{1}\right)=100-(i+1)$ and $V_{2}\left(T_{1}+T_{2}\right)=100-5 \times(i+1)$. The health state at any time point between $T_{1}$ and $T_{1}+T_{2}$ is found by linear interpolation. This health process reflects the fact that a shorter time to cancer relapse, $T_{2}$, may incur lower quality-of-life scores since, in general, it is considered to be a poor turn of events. For example, a patient whose gap time $T_{2}$ is between 36 and 48 months has a score of 96 at time $T_{1}$ that decreases to 
Table 3. International Breast Cancer Study Group Trial V example. Estimates of the joint distribution functions $\hat{F}_{Q}\left(q_{1}, q_{2}\right)$ under scenarios $V_{1}($.$) and V_{2}($.$) , respectively, for both the short- and long-$ duration chemotherapy treatment arms. Standard errors of the estimates are shown in parentheses

\begin{tabular}{lcccc} 
& \multicolumn{2}{c}{ Short-duration chemotherapy } & \multicolumn{2}{c}{ Long-duration chemotherapy } \\
$\left(q_{1}, q_{2}\right)$ & Scenario $V_{1}()$. & Scenario $V_{2}()$. & Scenario $V_{1}()$. & Scenario $V_{2}()$. \\
$(12,12)$ & $0 \cdot 087(0 \cdot 014)$ & $0 \cdot 063(0 \cdot 012)$ & $0 \cdot 114(0 \cdot 011)$ & $0 \cdot 092(0 \cdot 010)$ \\
$(24,12)$ & $0 \cdot 185(0 \cdot 019)$ & $0 \cdot 121(0 \cdot 016)$ & $0 \cdot 178(0 \cdot 014)$ & $0 \cdot 138(0 \cdot 012)$ \\
$(36,12)$ & $0 \cdot 249(0 \cdot 021)$ & $0 \cdot 155(0 \cdot 018)$ & $0 \cdot 217(0 \cdot 015)$ & $0 \cdot 160(0 \cdot 013)$ \\
$(48,12)$ & $0 \cdot 272(0 \cdot 023)$ & $0 \cdot 159(0 \cdot 018)$ & $0 \cdot 254(0 \cdot 016)$ & $0 \cdot 183(0 \cdot 014)$ \\
$(12,24)$ & $0 \cdot 135(0 \cdot 017)$ & $0 \cdot 097(0 \cdot 015)$ & $0 \cdot 125(0 \cdot 012)$ & $0 \cdot 115(0 \cdot 011)$ \\
$(24,24)$ & $0 \cdot 266(0 \cdot 022)$ & $0 \cdot 201(0 \cdot 020)$ & $0 \cdot 206(0 \cdot 014)$ & $0 \cdot 181(0 \cdot 014)$ \\
$(36,24)$ & $0 \cdot 372(0 \cdot 025)$ & $0 \cdot 270(0 \cdot 022)$ & $0 \cdot 265(0 \cdot 016)$ & $0 \cdot 221(0 \cdot 015)$ \\
$(48,24)$ & $0 \cdot 414(0 \cdot 027)$ & $0 \cdot 288(0 \cdot 024)$ & $0 \cdot 329(0 \cdot 018)$ & $0 \cdot 254(0 \cdot 016)$
\end{tabular}

80 at time $T_{1}+T_{2}$. In contrast, a patient with $T_{2}$ of at least 72 but no more than 84 months has scores of 99 at $T_{1}$ and 95 at $T_{1}+T_{2}$. There is flexibility in the method to explore such possibilities for dependence between quality-of-life and the gap times.

The estimates $\hat{F}_{Q}\left(q_{1}, q_{2}\right)$ of the joint distribution of $\left(Q T_{1}, Q T_{2}\right)$ at $\left(q_{1}, q_{2}\right)$, where $q_{1}=12,24,36,48$ months and $q_{2}=12,24$ months, are presented in Table 3, with the short- and long-duration chemotherapy groups shown side-by-side. In scenario $V_{1}($.$) , the$ estimates for the short-duration chemotherapy group are higher than those for the longduration chemotherapy group in almost every case. For example, in the short-duration chemotherapy group, the joint probability of a $Q T_{1}$ period lasting at most 48 months followed by a $Q T_{2}$ period lasting at most 12 months is equal to $0 \cdot 272$. This probability is higher than the corresponding value 0.254 of the same event in the long-duration chemotherapy group. However, in scenario $V_{2}($.$) , the probability of the same event$ occurrence in the long-duration chemotherapy group is equal to $0 \cdot 183$, higher than the corresponding probability 0.159 in the short-duration chemotherapy group. In general, under scenario $V_{2}($.$) , the estimates \hat{F}_{Q}\left(q_{1}, q_{2}\right)$ at $q_{2}=12$ months are more often lower in the short-duration chemotherapy group compared to the long-duration chemotherapy group, but this tendency is reversed for $q_{2}=24$ months.

The conditional distribution $\hat{F}_{Q}^{2 \mid 1}\left(q_{2} \mid q_{1}\right)$ estimates of $Q T_{2}$ given $Q T_{1}$, at the same values of $\left(q_{1}, q_{2}\right)$ as before, are shown in Table 4 . For example, under scenario $V_{1}($.$) , the probability$ of $Q T_{2}$ lasting at most 24 months, given that $Q T_{1}$ lasted at most 36 months, is equal to 0.803 in the short-duration chemotherapy group, compared to 0.876 in the long-duration chemotherapy group. A similar outcome is observed under scenario $V_{2}($.$) , when the$ same quantities are equal to 0.584 and 0.731 , respectively. Furthermore, when a direct comparison of the estimates obtained under $V_{1}($.$) and V_{2}($.$) is desired, as seen from Table 4,$ $\hat{F}_{Q}^{2 \mid 1}\left(q_{2} \mid q_{1}\right)$ is always higher under $V_{1}($.$) than under V_{2}($.$) , in each chemotherapy group.$

Equally true for both therapy groups, for $q_{2}$ fixed, a lower value of $q_{1}$ is usually associated with a higher value of the conditional distribution estimate $\hat{F}_{Q}^{2 \mid 1}\left(q_{2} \mid q_{1}\right)$; that is, the longer $Q T_{1}$, the higher the chance of experiencing a prolonged $Q T_{2}$ period. For example, in the short-duration chemotherapy group under $V_{1}($.$) , if q_{2}=24$ months, the probability of $Q T_{2}$ being at least 24 months, given that $Q T_{1}$ was at most 36 months, is equal to $0 \cdot 197$, while the probability of $Q T_{2}$ being at least 24 months, given that $Q T_{1}$ was at most 48 months, is equal to $0 \cdot 211$. Still in the short-duration chemotherapy group, but under scenario $V_{2}($.), 
Table 4. International Breast Cancer Study Group Trial V example. Estimates of the conditional distribution functions $\hat{F}_{Q}^{2 \mid 1}\left(q_{2} \mid q_{1}\right)$ under scenarios $V_{1}($.$) and V_{2}($.$) , respectively, for both the short- and long-$ duration chemotherapy treatment arms. Standard errors of the estimates are shown in parentheses

\begin{tabular}{lcccc} 
& \multicolumn{2}{c}{ Short-duration chemotherapy } & \multicolumn{2}{c}{ Long-duration chemotherapy } \\
$\left(q_{1}, q_{2}\right)$ & Scenario $V_{1}()$. & Scenario $V_{2}()$. & Scenario $V_{1}()$. & Scenario $V_{2}()$. \\
$(12,12)$ & $0.520(0.036)$ & $0.375(0.030)$ & $0.851(0.033)$ & $0.685(0.029)$ \\
$(24,12)$ & $0.562(0.037)$ & $0.369(0.030)$ & $0.776(0.031)$ & $0.602(0.027)$ \\
$(36,12)$ & $0.538(0.036)$ & $0.334(0.033)$ & $0.717(0.030)$ & $0.529(0.026)$ \\
$(48,12)$ & $0.519(0.036)$ & $0.307(0.027)$ & $0.675(0.029)$ & $0.485(0.025)$ \\
$(12,24)$ & $0.807(0.044)$ & $0.578(0.037)$ & $0.934(0.034)$ & $0.861(0.033)$ \\
$(24,24)$ & $0.807(0.044)$ & $0.613(0.039)$ & $0.898(0.033)$ & $0.791(0.031)$ \\
$(36,24)$ & $0.803(0.044)$ & $0.584(0.038)$ & $0.876(0.033)$ & $0.731(0.030)$ \\
$(48,24)$ & $0.789(0.043)$ & $0.548(0.037)$ & $0.874(0.033)$ & $0.674(0.029)$
\end{tabular}

the probabilities presented above are equal to 0.416 and 0.452 , respectively. On the other hand, in the long-duration chemotherapy group, under $V_{1}($.$) the same probabilities are$ equal to $0 \cdot 124$ and $0 \cdot 126$, respectively, while under $V_{2}($.$) they are 0.269$ and $0 \cdot 326$, respectively.

The comparisons involving joint and conditional probabilities in the two chemotherapy groups or under the two scenarios are shown for illustrative purposes. Testing for statistically significant differences between the joint distributions involved will be reported elsewhere.

\section{DisCUSSION}

Although our methodology corrects dependent censoring caused by shifting the survival time-scale to the quality-of-life-adjusted time-scale, it does not address dependent censoring as measured through prognostic covariates influencing both gap times and censoring times. Our approach to this problem is mainly nonparametric and assumes that censoring is independent of the gap times and of the health process. In well-controlled clinical trials patient drop-out is limited and censoring occurs for administrative reasons, at the end of the study, making these assumptions very reasonable. The independent censoring assumption has been made by authors such as Wang \& Wells (1998), Wang \& Chang (1999) and Lin et al. (1999) in recurrent/successive events problems, and by Zhao \& Tsiatis $(1997,1999)$, among others, in problems involving quality-of-life-adjusted lifetimes. There was some indication of mild dependent censoring as measured through oestrogen receptor status and menopausal status that was not addressed in this research. Doubly-robust estimators can be constructed under a coarsening at random assumption (van der Laan \& Hubbard, 1999; van der Laan et al., 2002) when covariates such as these are available, and this will be the subject of further research.

In nonparametric estimation of the joint distribution of a prespecified number of gap times or the conditional distribution of the current gap time, given the previous gap time(s), it would be natural to explore quality-of-life adjustment of the previous gap times. It is plausible that groups that have experienced comparable previous gap-time histories at similar overall quality-of-life levels, but that are currently living at $100 \%$ or at $50 \%$ levels of quality-of-life, would have different prognoses, in terms of both the ensuing gap 
time duration and the quality-of-life level during that gap time. In this case, separate analyses of the two aspects would fail to reveal such subtle differences.

We plan to develop methods for testing for overall differences in the joint distributions of the quality-of-life-adjusted gap times in two or more groups, including sensitivity analyses for the choice of utility function.

\section{ACKNOWLEDGEMENT}

The authors thank the International Breast Cancer Study Group for the use of their data. The authors are grateful to the editor for invaluable editorial work and to the associate editor for comments and suggestions that have helped improve this paper.

\section{APPENDIX}

Asymptotic theory

Consistency of $\hat{H}_{Q}($.$) , which in turn implies consistency of \hat{F}_{Q}($.$) and \hat{F}_{Q}^{K \mid K-1}(. \mid$.$) , is shown along$ the lines of Lin et al. (1999) and Zhao \& Tsiatis (1997), if we write

$$
\begin{aligned}
\hat{H}_{Q}\left(q^{(K)}\right)-H_{Q}\left(q^{(K)}\right) & =\tilde{H}_{Q}\left(q^{(K)}\right)-H_{Q}\left(q^{(K)}\right)+\hat{H}_{Q}\left(q^{(K)}\right)-\widetilde{H}_{Q}\left(q^{(K)}\right) \\
& =n^{-1} \sum_{i=1}^{n}\left[\frac{A_{i}\left(q^{(K)}\right)}{G\left\{D_{i}\left(q_{K}\right)\right\}}-H_{Q}\left(q^{(K)}\right)\right]+n^{-1} \sum_{i=1}^{n} A_{i}\left(q^{(K)}\right) \frac{G\left\{D_{i}\left(q_{K}\right)\right\}-\hat{G}\left\{D_{i}\left(q_{K}\right)\right\}}{G\left\{D_{i}\left(q_{K}\right)\right\} \hat{G}\left\{D_{i}\left(q_{K}\right)\right\}} .
\end{aligned}
$$

The first term in (A1) converges to zero in probability as a sum of zero-mean independent and identically distributed terms. Note that $D_{i}\left(q_{K}\right)<L<\tau_{C}:=\sup \{t ; G(t)>0\}$ and $\hat{G}($.$) converges$ uniformly in probability to $G($.$) on \left[0, \tau_{C}\right)$. The second term in (A1) is bounded from above, in absolute value, by

$$
\frac{\sup \left\{|\hat{G}(u)-G(u)| ; u \leqslant \tau_{C}\right\}}{\widehat{G}\left(\tau_{C}\right) G\left(\tau_{C}\right)},
$$

and therefore it converges to zero in probability. This concludes the proof that $\hat{H}_{Q}($.$) is consistent.$ Furthermore, if we define $M^{c}(u)=I\left\{C \leqslant \min \left(u, Y_{K}\right)\right\}-\int_{0}^{u} I\left(\tilde{Y}_{K} \geqslant s\right) d \Lambda^{c}(s)$, then, based on Lemma 2.4 of Gill (1983), it follows that, for any $t \leqslant \max \left\{\tilde{Y}_{K j} j=1, \ldots, n\right\}$, we have

$$
\frac{G(t)-\hat{G}(t)}{G(t)}=\int_{0}^{t} \frac{\hat{G}(u-)}{G(u)} \frac{\sum_{j=1}^{n} d M_{j}^{c}(u)}{\sum_{j=1}^{n} I\left(\tilde{Y}_{K j} \geqslant u\right)} .
$$

Letting $t=D_{i}\left(q_{K}\right)$, one obtains that

$$
\frac{G\left\{D_{i}\left(q_{K}\right)\right\}-\widehat{G}\left\{D_{i}\left(q_{K}\right)\right\}}{G\left\{D_{i}\left(q_{K}\right)\right\}}=\int_{0}^{L} I\left\{D_{i}\left(q_{K}\right) \geqslant u\right\} \frac{\widehat{G}(u-)}{G(u)} \frac{\sum_{j=1}^{n} d M_{j}^{c}(u)}{\sum_{j=1}^{n} I\left(\tilde{Y}_{K j} \geqslant u\right)} .
$$

Note that

$$
\begin{aligned}
W_{Q}\left(q^{(K)}\right):= & n^{1 / 2}\left\{\hat{H}_{Q}\left(q^{(K)}\right)-H_{Q}\left(q^{(K)}\right)\right\} \\
= & n^{1 / 2}\left\{\tilde{H}_{Q}\left(q^{(K)}\right)-H_{Q}\left(q^{(K)}\right)\right\}+n^{1 / 2}\left\{\hat{H}_{Q}\left(q^{(K)}\right)-\widetilde{H}_{Q}\left(q^{(K)}\right)\right\} \\
= & n^{-1 / 2} \sum_{i=1}^{n}\left[\frac{A_{i}\left(q^{(K)}\right)}{G\left\{D_{i}\left(q_{K}\right)\right\}}-H_{Q}\left(q^{(K)}\right)\right] \\
& +n^{-1 / 2} \int_{0}^{L} n^{-1} \sum_{i=1}^{n}\left[I\left\{D_{i}\left(q_{K}\right) \geqslant u\right\} \frac{A_{i}\left(q^{(K)}\right)}{\hat{G}^{(K)}\left\{D_{i}\left(q_{K}\right)\right\}} \frac{\hat{G}(u-)}{G(u)}\right] \frac{\sum_{j=1}^{n} d M_{j}^{c}(u)}{n^{-1} \sum_{j=1}^{n} I\left\{\tilde{Y}_{K j} \geqslant u\right\}} .
\end{aligned}
$$


Since

$$
\frac{\hat{G}(u-)}{G(u)} \frac{1}{n^{-1} \sum_{j=1}^{n} I\left(\tilde{Y}_{K j} \geqslant u\right)} \rightarrow\left\{\operatorname{pr}\left(\tilde{Y}_{K} \geqslant u\right)\right\}^{-1}
$$

almost surely, the second term in (A2) can further be expressed as

$$
\begin{aligned}
n^{-1 / 2} \int_{0}^{L} n^{-1} \sum_{i=1}^{n}\left[\left\{\operatorname { p r } \left(\tilde{Y}_{K}\right.\right.\right. & \left.\geqslant u)\}^{-1} I\left\{D_{i}\left(q_{K}\right) \geqslant u\right\} \frac{A_{i}\left(q^{(K)}\right)}{\widehat{G}\left\{D_{i}\left(q_{K}\right)\right\}}\right] \sum_{j=1}^{n} d M_{j}^{c}(u)+o_{P}(1) \\
& =n^{-1 / 2} \int_{0}^{L}\left\{\operatorname{pr}\left(\tilde{Y}_{K} \geqslant u\right)\right\}^{-1} E\left[I\left\{D\left(q_{K}\right) \geqslant u\right\} \frac{A\left(q^{(K)}\right)}{\widehat{G}\left\{D\left(q_{K}\right)\right\}}\right] \sum_{j=1}^{n} d M_{j}^{c}(u)+o_{P}(1) \\
& =n^{-1 / 2} \sum_{i=1}^{n} \int_{0}^{L}\left\{\operatorname{pr}\left(\tilde{Y}_{K} \geqslant u\right)\right\}^{-1} E\left[I\left\{D\left(q_{K}\right) \geqslant u\right\} \frac{A\left(q^{(K)}\right)}{G\left\{D\left(q_{K}\right)\right\}}\right] d M_{i}^{c}(u)+o_{P}(1) \\
& =n^{-1 / 2} \sum_{i=1}^{n} \int_{0}^{L} \frac{J_{Q}\left(q^{(K)}, u\right)}{\operatorname{pr}\left(\tilde{Y}_{K} \geqslant u\right)} d M_{i}^{c}(u)+o_{P}(1),
\end{aligned}
$$

where

$$
J_{Q}\left(q^{(K)}, u\right):=E\left[I\left\{D\left(q_{K}\right) \geqslant u\right\} \frac{A\left(q^{(K)}\right)}{G\left\{D\left(q_{K}\right)\right\}}\right]
$$

Consequently,

$$
W_{Q}\left(q^{(K)}\right)=n^{-1 / 2} \sum_{i=1}^{n}\left[\frac{A_{i}\left(q^{(K)}\right)}{G\left\{D_{i}\left(q_{K}\right)\right\}}-H_{Q}\left(q^{(K)}\right)\right]+n^{-1 / 2} \sum_{i=1}^{n} \int_{0}^{L} \frac{J_{Q}\left(q^{(K)}, u\right)}{\operatorname{pr}\left(\tilde{Y}_{K} \geqslant u\right)} d M_{i}^{c}(u)+o_{P}(1) .
$$

Hence, asymptotically,

$$
\begin{aligned}
\operatorname{cov}\left\{W_{Q}\left(q^{(K)}\right), W_{Q}\left(r^{(K)}\right)\right\}= & E\left(\left[\frac{A\left(q^{(K)}\right)}{G\left\{D\left(q_{K}\right)\right\}}-H_{Q}\left(q^{(K)}\right)+\int_{0}^{L} \frac{J_{Q}\left(q^{(K)}, u\right)}{\operatorname{pr}\left(\tilde{Y}_{K} \geqslant u\right)} d M^{c}(u)\right]\right. \\
& \left.\times\left[\frac{A\left(r^{(K)}\right)}{G\left\{D\left(r_{K}\right)\right\}}-H_{Q}\left(r^{(K)}\right)+\int_{0}^{L} \frac{J_{Q}\left(r^{(K)}, u\right)}{\operatorname{pr}\left(\widetilde{Y}_{K} \geqslant u\right)} d M^{c}(u)\right]\right) \\
= & E\left(\left[\frac{A\left(q^{(K)}\right)}{G\left\{D\left(q_{K}\right)\right\}}-H_{Q}\left(q^{(K)}\right)\right]\left[\frac{A\left(r^{(K)}\right)}{G\left\{D\left(r_{K}\right)\right\}}-H_{Q}\left(r^{(K)}\right)\right]\right) \\
& +E\left(\left[\frac{A\left(q^{(K)}\right)}{G\left\{D\left(q_{K}\right)\right\}}-H_{Q}\left(q^{(K)}\right)\right] \int_{0}^{L} \frac{J_{Q}\left(r^{(K)}, u\right)}{\operatorname{pr}\left(\tilde{Y}_{K} \geqslant u\right)} d M^{c}(u)\right) \\
& +E\left(\left[\frac{A\left(r^{(K)}\right)}{G\left\{D\left(r_{K}\right)\right\}}-H_{Q}\left(r^{(K)}\right)\right] \int_{0}^{L} \frac{J_{Q}\left(q^{(K)}, u\right)}{\operatorname{pr}\left(\widetilde{Y}_{K} \geqslant u\right)} d M^{c}(u)\right) \\
& +E\left\{\int_{0}^{L} \frac{J_{Q}\left(q^{(K)}, u\right)}{\operatorname{pr}\left(\tilde{Y}_{K} \geqslant u\right)} d M^{c}(u) \int_{0}^{L} \frac{J_{Q}\left(r^{(K)}, u\right)}{\operatorname{pr}\left(\tilde{Y}_{K} \geqslant u\right)} d M^{c}(u)\right\} .
\end{aligned}
$$

We can observe that (A3) is equal to

$$
E\left[\frac{A\left(q^{(K)}\right)}{G\left\{D\left(q_{K}\right)\right\}} \int_{0}^{L} \frac{J_{Q}\left(r^{(K)}, u\right)}{\operatorname{pr}\left(\tilde{Y}_{K} \geqslant u\right)} d M^{c}(u)\right]-E\left\{\int_{0}^{L} H_{Q}\left(q^{(K)}\right) \frac{J_{Q}\left(r^{(K)}, u\right)}{\operatorname{pr}\left(\tilde{Y}_{K} \geqslant u\right)} d M^{c}(u)\right\} .
$$

Since it represents the expected value of a zero-mean martingale, the second term in (A6) is equal to zero. The first term in (A6) and the expression in (A4) are both equal to

$$
-\int_{0}^{L} \frac{J_{Q}\left(q^{(K)}, u\right) J_{Q}\left(r^{(K)}, u\right)}{\operatorname{pr}\left(\tilde{Y}_{K} \geqslant u\right)} d \Lambda^{c}(u)
$$


based on arguments along the lines of Lin et al. (1999). Using standard results for stochastic integrals with respect to martingales, it follows that the expression in (A5) is equal to

$$
\begin{aligned}
E\left\{\int_{0}^{L} \frac{J_{Q}\left(q^{(K)}, u\right) J_{Q}\left(r^{(K)}, u\right)}{\left\{\operatorname{pr}\left(\tilde{Y}_{K} \geqslant u\right)\right\}^{2}} d\left\langle M^{c}(u), M^{c}(u)\right\rangle\right\} & =E\left\{\int_{0}^{L} \frac{J_{Q}\left(q^{(K)}, u\right) J_{Q}\left(r^{(K)}, u\right)}{\left\{\operatorname{pr}\left(\tilde{Y}_{K} \geqslant u\right)\right\}^{2}} I\left(\tilde{Y}_{K} \geqslant u\right) d \Lambda^{c}(u)\right\} \\
& =\int_{0}^{L} \frac{J_{Q}\left(q^{(K)}, u\right) J_{Q}\left(r^{(K)}, u\right)}{\operatorname{pr}\left(\widetilde{Y}_{K} \geqslant u\right)} d \Lambda^{c}(u) .
\end{aligned}
$$

By the multivariate central limit theorem, $W_{Q}($.$) converges in finite-dimensional distribution to a$ $K$-variate zero-mean Gaussian process with covariance function

$$
\begin{aligned}
\sigma_{H_{Q}}\left(q^{(K)} ; r^{(K)}\right)= & E\left(\left[\frac{A\left(q^{(K)}\right)}{G\left\{D\left(q_{K}\right)\right\}}-H_{Q}\left(q^{(K)}\right)\right]\left[\frac{A\left(r^{(K)}\right)}{G\left\{D\left(r_{K}\right)\right\}}-H_{Q}\left(r^{(K)}\right)\right]\right) \\
& -\int_{0}^{L} \frac{J_{Q}\left(q^{(K)}, u\right) J_{Q}\left(r^{(K)}, u\right)}{\operatorname{pr}\left(\tilde{Y}_{K} \geqslant u\right)} d \Lambda^{c}(u) .
\end{aligned}
$$

Recall that $F_{Q}\left(q^{(K)}\right)=H_{Q}\left(q_{0}^{(K)}\right)-H_{Q}\left(q^{(K)}\right)$, which can hence be estimated by $\hat{F}_{Q}\left(q^{(K)}\right)=$ $\hat{H}_{Q}\left(q_{0}^{(K)}\right)-\hat{H}_{Q}\left(q^{(K)}\right)$. Based on the previous results, it follows that $n^{-1 / 2}\left\{\hat{F}_{Q}()-.F_{Q}().\right\}$ converges in finite-dimensional distribution to a $K$-variate zero-mean Gaussian process with covariance function

$$
\begin{aligned}
\sigma_{F_{Q}}\left(q^{(K)} ; r^{(K)}\right)= & E\left(\left[\frac{A\left(q_{0}^{(K)}\right)}{G\{D(0)\}}-H_{Q}\left(q_{0}^{(K)}\right)-\frac{A\left(q^{(K)}\right)}{G\left\{D\left(q_{K}\right)\right\}}+H_{Q}\left(q^{(K)}\right)\right]\right. \\
& \left.\times\left[\frac{A\left(r_{0}^{(K)}\right)}{G\{D(0)\}}-H_{Q}\left(r_{0}^{(K)}\right)-\frac{A\left(r^{(K)}\right)}{G\left\{D\left(r_{K}\right)\right\}}+H_{Q}\left(r^{(K)}\right)\right]\right) \\
& -\int_{0}^{L} \frac{\left\{J_{Q}\left(q_{0}^{(K)}, u\right)-J_{Q}\left(q^{(K)}, u\right)\right\}\left\{J_{Q}\left(r_{0}^{(K)}, u\right)-J_{Q}\left(r^{(K)}, u\right)\right\}}{\operatorname{pr}\left(\tilde{Y}_{K} \geqslant u\right)} d \Lambda^{c}(u) .
\end{aligned}
$$

Next, we describe the asymptotic behaviour of $\hat{F}_{Q}^{K \mid K-1}\left(q_{K} \mid q^{(K-1)}\right)=1-\hat{H}_{Q}\left(q^{(K)}\right) / \hat{H}_{Q}\left(q_{0}^{(K)}\right)$, which estimates $F_{Q}^{K \mid K-1}\left(q_{K} \mid q^{(K-1)}\right)=1-H_{Q}\left(q^{(K)}\right) / H_{Q}\left(q_{0}^{(K)}\right)$. Define

$$
\begin{gathered}
U_{Q}\left(q^{(K)}\right):=n^{1 / 2}\left\{\hat{F}_{Q}^{K \mid K-1}\left(q_{K} \mid q^{(K-1)}\right)-F_{Q}^{K \mid K-1}\left(q_{K} \mid q^{(K-1)}\right)\right\} \\
=\frac{1}{H_{Q}\left(q_{0}^{(K)}\right)}\left[\left\{1-F_{Q}^{K \mid K-1}\left(q_{K} \mid q^{(K-1)}\right)\right\} n^{1 / 2}\left\{\hat{H}_{Q}\left(q_{0}^{(K)}\right)-H_{Q}\left(q_{0}^{(K)}\right)\right\}\right. \\
\left.\quad-n^{1 / 2}\left\{\hat{H}_{Q}\left(q^{(K)}\right)-H_{Q}\left(q^{(K)}\right)\right\}\right] .
\end{gathered}
$$

Based on the $W_{Q}($.$) convergence results, after computations it follows that U_{Q}($.$) converges in$ finite-dimensional distribution to a $K$-variate zero-mean Gaussian process with covariance function

$$
\sigma_{F_{Q}^{K \mid K-1}}\left(q_{K} ; q_{K}^{\prime} \mid q^{(K-1)}\right)=\frac{1}{H_{Q}^{2}\left(q_{0}^{(K)}\right)}\left[E\left\{R\left(q^{(K)}\right) R\left(q^{\prime(K)}\right)\right\}-\int_{0}^{L} \frac{S\left(q^{(K)}, u\right) S\left(q^{\prime(K)}, u\right)}{\operatorname{pr}\left(\tilde{Y}_{K} \geqslant u\right)} d \Lambda^{c}(u)\right],
$$

where

$$
\begin{gathered}
R\left(q^{(K)}\right)=\left\{1-F_{Q}^{K \mid K-1}\left(q_{K} \mid q^{(K-1)}\right)\right\}\left[\frac{A\left(q_{0}^{(K)}\right)}{G\{D(0)\}}-H_{Q}\left(q_{0}^{(K)}\right)\right]-\frac{A\left(q^{(K)}\right)}{G\left\{D\left(q_{K}\right)\right\}}+H_{Q}\left(q^{(K)}\right), \\
S\left(q^{(K)}, u\right)=\left\{1-F_{Q}^{K \mid K-1}\left(q_{K} \mid q^{(K-1)}\right)\right\} J_{Q}\left(q_{0}^{(K)}, u\right)-J_{Q}\left(q^{(K)}, u\right) .
\end{gathered}
$$


Estimators of all the previously presented covariance expressions are obtained as follows. The cumulative hazard $\Lambda^{c}(t)$ of the censoring distribution could be estimated by its Nelson-Aalen estimator

$$
\hat{\Lambda}^{c}(t)=\int_{0}^{t} \frac{\sum_{i=1}^{n}\left(1-\Delta_{K i}\right) d I\left(\tilde{Y}_{K i} \leqslant u\right)}{\sum_{i=1}^{n} I\left(\tilde{Y}_{K i} \geqslant u\right)} .
$$

The probability $\operatorname{pr}\left(\tilde{Y}_{K} \geqslant u\right)$ is consistently estimated by the sample mean $n^{-1} \sum_{i=1}^{n} I\left(\tilde{Y}_{K i} \geqslant u\right)$, while $J_{Q}\left(q^{(K)}, u\right)$ is estimated by

$$
\widehat{J}_{Q}\left(q^{(K)}, u\right)=n^{-1} \sum_{i=1}^{n} I\left\{D_{i}\left(q_{K}\right) \geqslant u\right\} \frac{B_{i}\left(q^{(K)}\right)}{\hat{G}\left\{D_{i}\left(q_{K}\right)\right\}} .
$$

In addition, $R_{i}\left(q^{(K)}\right)$ and $S\left(q^{(K)}, u\right)$ are estimated by

$$
\begin{gathered}
\hat{R}_{i}\left(q^{(K)}\right)=\left\{1-\hat{F}_{Q}^{K \mid K-1}\left(q_{K} \mid q^{(K-1)}\right)\right\}\left[\frac{B_{i}\left(q_{0}^{(K)}\right)}{\hat{G}_{\{}\left(D_{i}(0)\right\}}-\hat{H}_{Q}\left(q_{0}^{(K)}\right)\right]-\frac{B_{i}\left(q^{(K)}\right)}{\hat{G}^{(K)}\left(D_{i}\left(q_{K}\right)\right\}}+\hat{H}_{Q}\left(q^{(K)}\right), \\
\hat{S}\left(q^{(K)}, u\right)=\left\{1-\hat{F}_{Q}^{K \mid K-1}\left(q_{K} \mid q^{(K-1)}\right)\right\} \hat{J}_{Q}\left(q_{0}^{(K)}, u\right)-\hat{J}_{Q}\left(q^{(K)}, u\right) .
\end{gathered}
$$

Thus

$$
\begin{aligned}
& \hat{\sigma}_{H_{Q}}\left(q^{(K)} ; r^{(K)}\right)=n^{-1} \sum_{i=1}^{n}\left[\left(\frac{B_{i}\left(q^{(K)}\right)}{\hat{G}\left\{D_{i}\left(q_{K}\right)\right\}}-\hat{H}_{Q}\left(q^{(K)}\right)\right)\left(\frac{B_{i}\left(r^{(K)}\right)}{\hat{G}_{\{}\left\{D_{i}\left(r_{K}\right)\right\}}-\hat{H}_{Q}\left(r^{(K)}\right)\right)\right] \\
& -\int_{0}^{L} \frac{\widehat{J}_{Q}\left(q^{(K)}, u\right) \hat{J}_{Q}\left(r^{(K)}, u\right)}{n^{-1} \sum_{i=1}^{n} I\left(\tilde{Y}_{K i} \geqslant u\right)} d \hat{\Lambda}^{c}(u), \\
& \hat{\sigma}_{F_{Q}}\left(q^{(K)} ; r^{(K)}\right)=n^{-1} \sum_{i=1}^{n}\left[\frac{B_{i}\left(q_{0}^{(K)}\right)}{\hat{G}\left\{D_{i}(0)\right\}}-\hat{H}_{Q}\left(q_{0}^{(K)}\right)-\frac{B_{i}\left(q^{(K)}\right)}{\hat{G}_{\{}\left\{D_{i}\left(q_{K}\right)\right\}}+\hat{H}_{Q}\left(q^{(K)}\right)\right] \\
& \times\left[\frac{B_{i}\left(r_{0}^{(K)}\right)}{\widehat{G}\left\{D_{i}(0)\right\}}-\hat{H}_{Q}\left(r_{0}^{(K)}\right)-\frac{B_{i}\left(r^{(K)}\right)}{\widehat{G}\left\{D_{i}\left(r_{K}\right)\right\}}+\hat{H}_{Q}\left(r^{(K)}\right)\right] \\
& -\int_{0}^{L} \frac{\left\{\hat{J}_{Q}\left(q_{0}^{(K)}, u\right)-\hat{J}_{Q}\left(q^{(K)}, u\right)\right\}\left\{\hat{J}_{Q}\left(r_{0}^{(K)}, u\right)-\hat{J}_{Q}\left(r^{(K)}, u\right)\right\}}{n^{-1} \sum_{i=1}^{n} I\left(\tilde{Y}_{K i} \geqslant u\right)} d \hat{\Lambda}^{c}(u), \\
& \hat{\sigma}_{F_{Q}^{K \mid K-1}}\left(q_{K} ; q_{K}^{\prime} \mid q^{(K-1)}\right)=\frac{1}{\hat{H}_{Q}^{2}\left(q_{0}^{(K)}\right)}\left\{n^{-1} \sum_{i=1}^{n} \hat{R}_{i}\left(q^{(K)}\right) \hat{R}_{i}\left(q^{\prime(K)}\right)-\int_{0}^{L} \frac{\hat{S}\left(q^{(K)}, u\right) \hat{S}\left(q^{\prime(K)}, u\right)}{n^{-1} \sum_{i=1}^{n} I\left(\tilde{Y}_{K i} \geqslant u\right)} d \hat{\Lambda}^{c}(u)\right\},
\end{aligned}
$$

are consistent estimators of $\sigma_{H_{Q}}\left(q^{(K)} ; r^{(K)}\right), \sigma_{F_{Q}}\left(q^{(K)} ; r^{(K)}\right)$ and $\sigma_{F_{Q}^{K \mid K-1}}\left(q_{K} ; q_{K}^{\prime} \mid q^{(K-1)}\right)$, respectively.

\section{REFERENCES}

Gelber, R. D., Gelman, R. S. \& Goldhirsch, A. (1989). A quality-of-life oriented endpoint for comparing therapies. Biometrics 45, 781-95.

Gelber, R. D., Goldhirsch, A., Hurny, C., Bernhard, J. \& Simes, R. J. for the International Breast Cancer Study Group (IBCSG) (1992). Quality of life in clinical trials of adjuvant therapies. J. Nat. Cancer Inst. Monog. 11, 127-35.

GiLl, R. D. (1983). Large sample behaviour of the product-limit estimator on the whole line. Ann. Statist. 11, 49-58.

Huang, Y. (2000). Two-sample multistate accelerated sojourn times model. J. Am. Statist. Assoc. 95, 619-27.

Huang, Y. \& Louis, T. A. (1998). Nonparametric estimation of the joint distribution of survival time and mark variables. Biometrika 85, 785-98.

HuANG, Y. \& Louis, T. A. (1999). Expressing estimators of expected quality adjusted survival as functions of Nelson-Aalen estimators. Lifetime Data Anal. 5, 199-212.

LIN, D. Y., Sun, W. \& YING, Z. (1999). Nonparametric estimation of the gap time distributions for serial events with censored data. Biometrika 86, 59-70. 
Peña, E. A., Strawderman, R. L. \& Hollander, M. (2001). Nonparametric estimation with recurrent event data. J. Am. Statist. Assoc. 96, 1299-315.

Robins, J. M. \& Rotnitzky, A. (1992). Recovery of information and adjustment for dependent censoring using surrogate markers. In Proc. Am. Statist. Assoc., AIDS Epidemiology-Methodological Issues, Ed. N. Jewell, K. Dietz and V. Farewell, pp. 297-331. Alexandria, VA: American Statistical Association.

Van Der LaAn, M. J. \& Hubbard, A. E. (1999). Locally efficient estimation of the quality-adjusted lifetime distribution with right-censored data and covariates. Biometrics 55, 530-6.

Van Der Laan, M. J., Hubbard, A. E. \& Robins, J. M. (2002). Locally efficient estimation of a multivariate survival function in longitudinal studies. J. Am. Statist. Assoc. 97, 494-507.

Wang, M. C. \& Chang, S. H. (1999). Nonparametric estimation of a recurrent survival function. J. Am. Statist. Assoc. 94, 146-53.

WANG, M. C., QIN, J. \& ChIANG, C. T. (2001). Analyzing recurrent event data with informative censoring. J. Am. Statist. Assoc. 96, 1057-65.

Wang, W. \& Wells, M. T. (1998). Nonparametric estimation of successive duration times under dependent censoring. Biometrika 85, 561-72.

ZhaO, H. \& Tsiatis, A. A. (1997). A consistent estimator for the distribution of quality adjusted survival time. Biometrika 84, 339-48.

Zhao, H. \& Tsiatis, A. A. (1999). Efficient estimation of the distribution of quality-adjusted survival time. Biometrics 55, 1101-7.

[Received February 2005. Revised October 2005] 